\title{
Faecal unconjugated bile acids in patients with colorectal cancer or polyps
}

\author{
C H E Imray, S Radley, A Davis, G Barker, C W Hendrickse, I A Donovan, A M Lawson, \\ P R Baker, J P Neoptolemos
}

\begin{abstract}
The unconjugated faecal bile acid profiles of 14 patients with colorectal cancer, nine patients with polyps and 10 controls were compared using gas liquid chromatography, controlling for such confounding variables as cholecystectomy, gall stones and hepatic function. Patients with adenomatous polyps had a higher concentration of faecal bile acids $(5.23 \mu \mathrm{mol} /$ g, 2.16-13.67 (median, range) $v 1.96,0.91-$ $6.97 ; \mathrm{p}=0.016)$ lithocholic acid $(2.41,0.88$ $3.22 v 1.07,0.38-2.03 ; \mathrm{p}=0.013)$ and total secondary bile acids $(5 \cdot 23,2 \cdot 16-13.4 v 1.96$, 0.73-6.63; $p=0.02$ ) compared with control subjects. Patients with colorectal cancer had an increased $(p=0.029)$ proportion of secondary faecal bile acids (mol\%) compared with controls $(100,96 \cdot 5-100 v 95 \cdot 19,81 \cdot 73-100)$ and the ratios of the primary bile acids, cholic and chenodeoxycholic acid, to their respective derivatives (secondary bile acids) were significantly lower in cancer patients compared with control and patients with polyps $(p=0.034$ to 0.004$)$. This study lends further support to the theory that bile acids may play a role in the development of polyps and colorectal cancer.
\end{abstract}

(Gut 1992; 33: 1239-1245)

Epidemiological studies have implicated dietary fat of animal origin as an important aetiological factor in the development of colorectal cancer ${ }^{2}$ which may be related to altered faecal bile acids. ${ }^{3}$ Bile acids given either orally, ${ }^{4}$ or rectally, ${ }^{56}$ have been shown to have a tumour promoting effect in rat models for colorectal cancer.

The mechanisms by which bile acids may produce these effects are uncertain. Bile acids may be mutagenic, ${ }^{78}$ but some of the techniques used have been questioned. ${ }^{9-11}$ Bile acids may directly damage DNA, ${ }^{12}$ or chromatin. ${ }^{13}$ Lithocholic acid has been shown to produce DNA strand breaks ${ }^{14}$ and both chenodeoxycholic acid and lithocholic acid induce mitotic aneuploidy in yeast cells. ${ }^{15}$ Possible mechanisms which may explain an increase in colonic cell proliferation caused by bile acids, include effects on prostaglandin synthesis, ${ }^{16}$ arachidonic acid release, ${ }^{17}$ and protein kinase $\mathrm{C}$ activity. ${ }^{18}$

Populations at greater risk of colorectal cancer have been found to excrete more faecal bile acids, ${ }^{31920}$ but others have not shown this. ${ }^{21}$ The correlation between dietary habits and faecal bile acid excretion, is also supported by differences found in the faecal bile acid profiles of certain population subgroups, ${ }^{2022}$ or different socioeconomic groups. ${ }^{23}$ Furthermore, dietary manipulation studies have shown that high fat intake can increase faecal bile acid excretion ${ }^{2+25}$

Two early studies showed an increased faecal bile acid excretion in patients with colonic cancer, ${ }^{2627}$ but later studies have been unable to confirm this. ${ }^{28-3+}$ Only one of four studies in polyps has shown any increase in faecal bile acid excretion in this group. ${ }^{26313536}$ Differences in patient selection, faecal collection, and analytical methodology may account for these apparently conflicting results. ${ }^{21}$

More recently Hill and Owen, unable to show their previous findings of a significant increase in faecal bile acid excretion, have proposed instead that the ratio of lithocholic acid: deoxycholic acid and lithocholic acid: deoxycholic acid $\times$ total faecal bile acid concentration may be important markers in patients with colorectal cancer. ${ }^{37} 38$

The role of the colon in modifying biliary bile acid composition, ${ }^{39}$ has prompted several groups $^{40-43}$ to examine the biliary bile acid profiles of patients with colorectal cancer or adenomatous polyps with conflicting results. Again differences in analytical techniques, patient selection, and other variables makes the interpretation of some of these studies difficult.

In this study we report the results of detailed analysis of the faecal unconjugated bile acid profiles of patients with colorectal cancer or polyps in comparison with controls and in which a careful attempt at controlling for confounding factors has been made.

\section{Methods}

PATIENTS AND STUDY DESIGN

Patients with colorectal cancer, adenomatous polyps or controls were recruited from the general surgical outpatients' department of Dudley Road Hospital.

Patients with colorectal cancer (14) or adenomatous polyps (nine) entered the study after diagnosis. None of these patients were obstructed. The control group (10) comprised patients with non-specific abdominal symptoms other than constipation. Control subjects as well as patients with cancer or polyps underwent rigid sigmoidoscopy and colonoscopy or double contrast barium enema.

All groups underwent abdominal ultrasound scan to determine the presence of gall stones and exclude liver metastases in the cancer group, as well as biochemical tests to assess hepatic and renal function. Weight was measured and recorded for each patient. All were white caucasians on an ad libitum omnivorous diet.

Patients with abnormal liver function tests, gall stones, previous cholecystectomy, or hepatic metastases were excluded. No patient had a 
coexisting medical complaint likely to affect bile acid metabolism nor had any undergone previous bowel surgery other than appendicectomy. Any patients who had taken antibiotics within the three months before entering the study, or other medication likely to affect bile acid metabolism, were also excluded.

The histological examination of carcinomas and polyps was carried out by the Pathology Department of the Dudley Road Hospital.

\section{FAECAL COLLECTION}

Complete five day faecal collections were obtained from patients, at home or place of work and taking their normal diet. This was achieved by delivering small 481 (Frigidaire, FV 602) freezers to the homes of the participants so that stools could be frozen immediately at $-22^{\circ} \mathrm{C}$. At the same time patients carried out a mouth-toanus transit time study using radioopaque markers (Portex UK Ltd). ${ }^{+4}$ Subjects were asked to collect every stool for five days and record the time each was passed. After five days the freezers were collected and returned to the laboratory. Faecal samples were stored at $-20^{\circ} \mathrm{C}$ before being radiographed (for transit time studies), homogenisation and freeze drying.

\section{HOMOGENISATION}

Each five day stool collection was rapidly thawed and stools were weighed before the whole collection was pooled.

The pooled samples were homogenised for 20 minutes in five times the faecal weight of cold water using a Stomacher 3500 (Seward, London, UK). A portion of the homogenate was then transferred to a beaker containing a magnetic stirrer (to ensure continuous mixing) and $10 \mathrm{ml}$ aliquots were transferred to $20 \mathrm{ml}$ glass vials, freeze dried (Edwards Modulyo freeze drier with three shelf closing accessory) for four days (with heating after 24 hours) and sealed under vacuum. The lyophylised samples were stored until required for analysis.

\section{BILE ACID EXTRACTION}

Bile acids were extracted according to methods previously described by Setchell $e t a l,{ }^{4546}$ with a recent modification. ${ }^{47}$

Duplicate samples of $10 \mathrm{mg}$ freeze dried faeces were taken, $100 \mu \mathrm{l}^{3} \mathrm{H}$ cholic acid $(17 \mathrm{nCi}=0.68$ $\mathrm{pmol}$ ) and $0.25 \mu \mathrm{mol}$ nordeoxycholic acid were added as recovery standards.

The faecal sample was transferred to a $250 \mathrm{ml}$ round bottomed flask with $10 \mathrm{ml} 90 \%$ ethanol and sonicated for 15 minutes before addition of $20 \mathrm{ml}$ hexane. The flask was swirled and a bilayer allowed to form. The hexane (upper layer) was then decanted into a clean flask. The hexane step was repeated, the decanted hexane pooled, dried on a rotary evaporator and stored. A further 35 $\mathrm{ml} 90 \%$ ethanol was added to the flask containing the ethanolic faecal suspension, which was then refluxed gently for two hours. After reflux the sample was cooled on ice and centrifuged at $1500 \mathrm{~g}$ for 15 minutes. The supernatant was removed and saved. The faecal pellet was resus- pended in $45 \mathrm{ml} 80 \%$ ethanol and refluxed for a further two hours. The sample was centrifuged and the supernatant retained. The faecal pellet was then resuspended in $45 \mathrm{ml}$ chloroformmethanol $(1: 1 ; \mathrm{vol}: \mathrm{vol})$ and refluxed for a further one hour. Again the sample was centrifuged and the supernatant saved. Finally the pellet was resuspended in $45 \mathrm{ml}$ chloroform:methanol and sonicated (to minimise any losses) before being centrifuged and the supernatant collected. The extracted supernatants were pooled, in a clean round bottomed flask and dried using a rotary evaporator.

The dried sample was resuspended in $5 \mathrm{ml}$ acid water ( $\mathrm{pH} 3-4)$ which was then sonicated for five minutes. The suspension was then filtered through a $10 \times 1 \mathrm{~cm}$ column of Lipidex 1000 . The column was converted to the aqueous form with $40 \mathrm{ml}$ distilled water before use. The eluate was collected in a conical flask together with a column wash of $40 \mathrm{ml}$ water (aqueous extract). Bile acids were recovered from the Lipidex 1000 by eluting the column with $40 \mathrm{ml} 100 \%$ methanol into the original round bottomed flask.

The aqueous extract was then passed through a Sep Pak C18 cartridge $(500 \mathrm{mg}$ ) prepared by previous washing with $5 \mathrm{ml} 100 \%$ methanol followed by $10 \mathrm{ml}$ water. Bile acids were eluted from the cartridge with $5 \mathrm{ml} 100 \%$ methanol. The methanolic eluates were pooled in the original round bottomed flask and dried on a rotary evaporator.

The extracted bile acids were resuspended in $20 \mathrm{ml} 72 \%$ ethanol. The pooled 'hexane' fraction (stored earlier) was resuspended in $10 \mathrm{ml} 72 \%$ ethanol. The ethanolic suspensions were then filtered through a column of Amberlyst A-15 $(2 \mathrm{~cm} \times 10 \mathrm{~cm})$, the column then being washed with a further $20 \mathrm{ml}$ of $72 \%$ ethanol and the samples pooled. The column was prepared by suspending the Amberlyst A-15 in $72 \%$ ethanol for 24 hours before slurrying it into columns and allowing it to settle by gravity. This was then treated with $50 \mathrm{ml} 2 \mathrm{M} \mathrm{NaOH}$ in $72 \%$ ethanol and washed to neutrality with $200 \mathrm{ml} 72 \%$ ethanol. It was converted to the $\mathrm{H}^{+}$form by washing with $50 \mathrm{ml} 2 \mathrm{M} \mathrm{HCl}$ in $72 \%$ ethanol, followed by $72 \%$ ethanol until the eluate was neutral. After use, the Amberlyst was resuspended in $72 \%$ ethanol until required when it was reactivated as described. The Amberlyst resin was changed after every 10 extractions.

\section{GROUP SEPARATION}

Group separation was achieved by applying the effluent from the Amberlyst to a column of Lipidex DEAP (dietylaminohydroxypropyl Sephadex LH-20) as described by Almé et al. ${ }^{48}$ This was prepared in the acetate form by washing $100 \mathrm{~g}$ Lipidex-DEAP on a sintered glass funnel with $500 \mathrm{ml}$ potassium acetate- $\mathrm{KOH}$ $0.5 \mathrm{M}$ in $72 \%$ ethanol and then with further volumes of $72 \%$ ethanol until the eluate was neutral. It was then washed with $500 \mathrm{ml} 0 \cdot 1 \mathrm{M}$ acetic acid in $72 \%$ ethanol and washed to neutrality again with $72 \%$ ethanol. The gel was slurried into glass columns and allowed to settle by gravity ( $\approx 30$ minutes) to give a column size of $0.5 \times 14 \mathrm{~cm}$. After the addition of the sample, it 
was eluted under pressure using nitrogen $(0 \cdot 5-$ $1.0 \mathrm{Kg} \mathrm{cm}^{-2}$ ) to give a flow rate of $50-80$ drops per minute. The column was washed with $9 \mathrm{ml}$ $72 \%$ ethanol $(\mathrm{pH} \mathrm{7})$ to give a fraction comprising neutral compounds and any esterfied bile acids. Unconjugated bile acids were obtained by elution with $7.5 \mathrm{ml} 0.15 \mathrm{M}$ acetate in $72 \%$ ethanol (apparent $\mathrm{pH} 3 \cdot 8$, adjusted with ammonium hydroxide) followed by $4.5 \mathrm{ml} 72 \%$ ethanol. The remaining glycine conjugated, taurine conjugated and sulphated bile acid fractions were obtained by continued stepwise elutions with acetate buffers, but are not further considered in this report.

The unconjugated fractions were dried on a rotary evaporator and taken up in methanol as required.

\section{DERIVITISATION}

The internal standard $(62.5 \mathrm{nmol}$ coprostanol) was added to the sample. Diazomethane was freshly prepared using $0.54 \mathrm{~g} \mathrm{KOH}$ dissolved in $14 \mathrm{ml} 90 \%$ methanol and $2 \cdot 86 \mathrm{~g} \mathrm{~N}$-methyl-nnitroso-p-toluenesulphonamide in $40 \mathrm{ml}$ diethyl ether in a round bottom flask. After five minutes condensed diazomethane was collected in an ice cooled tube. The bile acid mixture was transferred to a screw top reaction vial containing $0.5 \mathrm{ml}$ methanol and $1 \mathrm{ml}$ diazomethane. Methylated bile acids were produced by reaction at room temperature for one hour and subsequently evaporated to dryness under nitrogen. The trimethylsilyl ester derivatives were obtained by reacting the dried bile acid sample with $200 \mu \mathrm{l}$ of the silanisation mixture (pyridine: hexamethyldisilazine : trimethylchlorosilane; 3:2:1; vol:vol:vol) at $55^{\circ} \mathrm{C}$ for one hour. The derivative was dried and extracted with $3 \times 200$ $\mu l$ hexane and centrifuged in a microtube at $13400 \mathrm{~g}$ for 10 minutes. The supernatant was transferred to a glass gas liquid chromatography vial (Chromacol Ltd, London, UK) dried and resuspended in $200 \mu$ l hexane.

\section{CHROMATOGRAPHY}

A $1 \mu$ l volume was injected onto the $25 \mathrm{~m} \mathrm{DB} 1$ capillary tube column with a $1 \mathrm{~m}$ retention gap (Jones Chromatography, Hengoed, Wales) of a PU4400 gas chromatography with a PU4700 auto injector (Phillips Scientific, Cambridge, UK). The sample had a 170:1 split, at an initial temperature of $220^{\circ} \mathrm{C}$, ramping at $2^{\circ} \mathrm{C} /$ minute to $280^{\circ} \mathrm{C}$ at which it was kept for 15 minutes, using helium as the carrier gas. Detection was by flame ionisation using hydrogen and air at $290^{\circ} \mathrm{C}$ together with helium as a make up gas to improve the detection limit. The integration system was a PU3202 computer utilising PU6000 software (Phillips Scientific). Ultra pure gases were used and were further purified before entry into the chromatograph using the following filters; oxyfilter, moisture filter and charcoal filter $\times 2$ (Chrompack, London, UK).

The lower detection limit for bile acids was $0 \cdot 3$ pmol. Putative bile acids were identified by comparing peaks with the retention times of authentic standards and subsequently confirmed by gas liquid chromatography mass spectro- metry ${ }^{49}$ at the Clinical Research Centre's Unit of Mass Spectrometry. Relative retention times and response factors were calculated by comparison of authentic standards to coprostanol. Concentrations of the individual bile acids were calculated by comparing their peak areas with that of the internal standard coprostanol. The values from at least two chromatograms were used to obtain a mean; the percentage individual bile acid present was calculated from the micromolar concentrations. Total bile acid concentration was calculated as the sum of all gas liquid chromatography detected individual bile acids.

\section{STATISTICAL ANALYSIS}

All results are expressed as the median value with the range to indicate dispersion.

Comparisons between the three groups was undertaken using the Kruskall-Wallis test adjusted for ties; if significant $(\mathrm{p}<0.05)$ subsequent analysis between two groups was undertaken using the two-tailed Mann-Whitney U test. A $\chi^{2}$ test was used to evaluate differences in sex distribution and frequency of occurrence of individual bile acids. Tests of correlation were undertaken using Spearman's rank correlation test (two-tailed). Significance was taken as $\mathrm{p}<0.05$.

\section{ETHICAL CONSIDERATIONS}

All patients gave informed consent. The study was approved by the Ethical Committee of the West Birmingham Health Authority.

\section{MATERIALS}

Reference bile acids were obtained from Steraloids Ltd (Croydon, UK) and Sigma UK Ltd (Poole, UK). ${ }^{3} \mathrm{H}$ cholic acid was obtained from Amersham International PLC (Aylesbury, UK). All solvents were AR grade and were obtained from Fisons along with pyridine (Loughborough, UK). All other reagents were purchased from Sigma UK Ltd. Lipidex-DEAP was obtained from Packard United Technologies (Pangbourne, UK). Sep-pak C-18 cartridges (500 mg) were purchased from Millipore-Waters (Watford, UK). All water used was double distilled and deionised using the Milli-Q system (Millipore, Watford, UK). Helium CP grade ( $>99.999 \%$ pure), Hydrogen hydrocarbon zero grade ( $>99.999 \%$ pure), and air BTCA 74 grade were supplied by BOC-Special Gases (Wolverhampton, UK).

\section{Results}

\section{PATIENTS}

There were no significant differences in the ages, weights, or stool frequency between the three groups. Transit times and daily stool weights are shown in Table I.

All the cancer patients had adenocarcinomas: two had Dukes's stage A, seven had B, and five had $C$. The polyps varied in size from $0.5 \mathrm{~cm}$ to $1.0 \mathrm{~cm}$ and five showed dysplastic changes (one mild and four moderate dysplasia). 
BILE ACIDS

The mean recovery of the radiolabelled standard was $92.9 \%(7 \cdot 11)(\mathrm{SD})$. A typical chromatogram is shown in the Figure; 16 unconjugated bile acids were identified during this study (Table II). From our own observations (unpublished) and those of others, ${ }^{45}$ sulphated and conjugated bile acids represent less than $10 \%$ of the total bile

TABLE I Patient details

\begin{tabular}{lccc}
\hline & $\begin{array}{c}\text { Controls } \\
(n=10)\end{array}$ & $\begin{array}{c}\text { Cancers } \\
(n=14)\end{array}$ & $\begin{array}{c}\text { Polyps } \\
(n=9)\end{array}$ \\
\hline Sex Male & 7 & 8 & $9^{\star}$ \\
Female & 3 & 6 & 0 \\
Age (yr) & $68 \cdot 5$ & $67 \cdot 5$ & 65 \\
Weight (kg) & $60-81$ & $47-79$ & $27-73$ \\
& $65 \cdot 5$ & 71 & $70 \cdot 5$ \\
Stools/day & $53-98$ & $44-92$ & $62-92$ \\
& $1 \cdot 3$ & $1 \cdot 7$ & $1 \cdot 8$ \\
Wet wt/day (g) & $0 \cdot 8-5$ & $0 \cdot 4-7$ & $1-2 \cdot 8$ \\
& $149^{\mathrm{a}}$ & $86^{\mathrm{b}}$ & $161^{\mathrm{c}}$ \\
Transit time (hr) & $81-308$ & $12-150$ & $94-337$ \\
& $68^{\mathrm{d}}$ & $114^{\mathrm{e}}$ & $52^{\mathrm{f}}$ \\
& $15-114$ & $42-114$ & $11-94$ \\
\hline
\end{tabular}

Upper figures indicate median, lower figures in italics indicate

${ }^{2}(\mathrm{df}=2) 0.1>\mathrm{p}>0.05$.

Kruskall-Wallis $(\mathrm{H}=12 \cdot 3, \mathrm{df}=2) \quad{ }^{\mathrm{a}} v^{\mathrm{b}} v^{\mathrm{c}} \mathrm{p}=0.002$

Kruskall-Wallis $(\mathrm{H}=10.7, \mathrm{df}=2) \quad \mathrm{d}_{v} \mathrm{e}^{\mathrm{f}} \mathrm{p}=0.005$

Mann Whitney ${ }^{\mathrm{a}} v^{\mathrm{b}} \mathrm{p}=0.007$

${ }^{\mathrm{b}} v^{\mathrm{c}} \mathrm{p}=0.002$

$v^{\mathrm{c}} \mathrm{p}=0.84$

$v^{\mathrm{e}} \mathrm{p}=0.033$

$\mathrm{d}^{\mathrm{f}} \mathrm{p}=0.002$
${ }_{\mathrm{p}}=0.31$

TABLE II Faecal bile acid concentration ( $\mu \mathrm{mol} / \mathrm{g}$ dry faeces) in control, cancer, and polyp patients

\begin{tabular}{|c|c|c|c|}
\hline Bile acid & $\begin{array}{l}\text { Control } \\
(n=10)\end{array}$ & $\begin{array}{l}\text { Cancer } \\
(n=14)\end{array}$ & $\begin{array}{l}\text { Polyp } \\
(n=9)\end{array}$ \\
\hline Lithocholate & $1 \cdot 07(10)^{\mathrm{a}}$ & \multirow{32}{*}{$\begin{array}{l}1 \cdot 56(14)^{\mathrm{b}} \\
0 \cdot 37-3 \cdot 83 \\
1 \cdot 17(14) \\
0 \cdot 09-2 \cdot 53 \\
0 \cdot 42(14) \\
0 \cdot 14-0 \cdot 8 \\
0 \cdot 13(11) \\
0-0 \cdot 52 \\
0 \cdot 18(10) \\
0-1 \cdot 61 \\
0 \cdot 06(9) \\
0-0 \cdot 36 \\
0 \cdot 00(4) \\
0-0 \cdot 12 \\
0 \cdot 00(1)^{\mathrm{e}} \\
0-0 \cdot 12 \\
0 \cdot 00(4) \\
0-0 \cdot 12 \\
0 \cdot 00(6) \\
0-0 \cdot 14 \\
0 \cdot 00(3) \\
0-0 \cdot 05 \\
0 \cdot 07(11) \\
0-0 \cdot 46 \\
0 \cdot 00(2) \\
0-0 \cdot 43 \\
0 \cdot 00(2) \\
0-0 \cdot 01 \\
0 \cdot 00(1) \\
0-0 \cdot 04 \\
0 \cdot 00(1) \\
0-0 \cdot 06 \\
3 \cdot 93^{\mathrm{h}} \\
0 \cdot 87-8 \cdot 29\end{array}$} & $2.41(9)^{\mathrm{c}}$ \\
\hline $\begin{array}{l}3 \alpha \text { hydroxy- } 5 \beta \text { cholanoate } \\
\text { Deoxycholate }\end{array}$ & $\begin{array}{l}0.38-2 \cdot 03 \\
0.61(10)\end{array}$ & & $0 \cdot 88-3 \cdot 22$ \\
\hline $3 \alpha, 12 \alpha$ dihydroxy- $5 \beta$ cholanoate & $\begin{array}{l}0.01(10) \\
0.18-2 \cdot 78\end{array}$ & & $\begin{array}{l}1 \cdot 33(9) \\
0.8-6 \cdot 89\end{array}$ \\
\hline $\begin{array}{l}\text { Isolithocholate } \\
\text { S }\end{array}$ & $0.35(10)$ & & $0.42(9)$ \\
\hline $3 \beta$ hydroxy $-5 \beta$ cholanoate & $0.07-1.03$ & & $0.18-0.96$ \\
\hline Trans-isodeoxycholate & $0.07(9)$ & & $0.24(9)$ \\
\hline $3 \alpha, 12 \beta$ dihydroxy- $5 \alpha$ cholanoate & $0-0.36$ & & $0.06-0.69$ \\
\hline 12-oxo-lithocholate & $0.09(7)$ & & $0 \cdot 14(7)$ \\
\hline $3 \alpha$ hydroxy-120xo- $5 \beta$ cholanoate & $0-0.35$ & & $0-0.82$ \\
\hline Isodeoxycholate & $0.03(6)$ & & $0 \cdot 23(8)$ \\
\hline $3 \alpha, 12 \beta$ dihydroxy $-5 \beta$ cholanoate & $0-0 \cdot 18$ & & $0-0.49$ \\
\hline Trans-isolithocholate & $0.00(4)$ & & $0.00(3)$ \\
\hline $3 \beta$ hydroxy $-5 \alpha$ cholanoate & $0-0 \cdot 17$ & & $0-0 \cdot 2$ \\
\hline Chenodeoxycholate & $0.05(6)^{d}$ & & $0.07(5)^{f}$ \\
\hline $3 \alpha, 7 \alpha$ dihydroxy- $5 \beta$ cholanoate & $0-0 \cdot 23$ & & $0-0.78$ \\
\hline Cholate & $0.05(6)$ & & $0 \cdot 1(6)$ \\
\hline $3 \alpha, 7 \alpha, 12 \alpha$ trihydroxy- $5 \beta$ cholanoate & $0-0 \cdot 14$ & & $0-1.06$ \\
\hline Hyodeoxycholate & $0.00(4)$ & & $0.00(2)$ \\
\hline $3 \alpha, 6 \alpha$ dihydroxy- $5 \beta$ cholanoate & $0-0 \cdot 15$ & & $0-0.05$ \\
\hline Ursodeoxycholate & $0.00(4)$ & & $0.00(4)$ \\
\hline $3 \alpha 7 \beta$ dihydroxy- $5 \beta$ cholanoate & $0-0.24$ & & $0-0.87$ \\
\hline & $0.00(4)$ & & $0 \cdot 1(6)$ \\
\hline $3 \alpha, 7 \beta, 12 \beta$ trihydroxy- $5 \beta$ cholanoate & $0-0 \cdot 22$ & & $0-3 \cdot 37$ \\
\hline 7-oxo-lithocholate & $0.00(2)$ & & $0.00(1)$ \\
\hline $3 \alpha$ hydroxy-7oxo- $5 \beta$ cholanoate & $0-0.32$ & & $0-1.02$ \\
\hline 7-oxo-deoxycholate & & & \\
\hline $3 \alpha, 12 \alpha$ dihydroxy-7oxo- $5 \beta$ cholanoate & $N D$ & & ND \\
\hline 12-oxo-chenodeoxycholate & $0.00(1)$ & & \\
\hline $3 \alpha, 7 \alpha$ dihydroxy- $5 \beta$ cholanoate & $0-0 \cdot 1$ & & $N D$ \\
\hline \multirow{3}{*}{$\begin{array}{l}3 \alpha \text { hydroxy- } 7,12 \text { dioxo- } 5 \beta \text { cholanoate } \\
\text { SUM } 2^{\circ} \text { BA }\end{array}$} & $N D$ & & $N D$ \\
\hline & $1.96^{8}$ & & $5 \cdot 23^{i}$ \\
\hline & $0 \cdot 74-6 \cdot 63$ & & $2 \cdot 16-13 \cdot 40$ \\
\hline
\end{tabular}

Upper figures indicate median values, lower figures in italics indicate range, figures in brackets indicate number of patients in which bile acid detected. $\mathrm{ND}=$ not detected.

Frequency of detection of chenodeoxycholate: ${ }^{\mathrm{d}} v^{\mathrm{e}} v^{\mathrm{f}} \chi^{2}(\mathrm{df}=2) 0.02>\mathrm{p}>0.01$

Kruskall-Wallis $(\mathrm{H}=6.77, \mathrm{df}=2) \quad{ }^{\mathrm{a}} v^{\mathrm{b}} v^{\mathrm{c}} \mathrm{p}=0.034$

Kruskall-Wallis $(\mathrm{H}=8.54, \mathrm{df}=2) \quad \mathrm{d}^{\mathrm{e}} v^{\mathrm{f}} \mathrm{p}=0.014$

Kruskall-Wallis $(\mathrm{H}=6.33, \mathrm{df}=2) \quad \mathrm{g} v^{\mathrm{h}} v^{\mathrm{i}} \mathrm{p}=0.043$

Mann Whitney ${ }_{\mathrm{a}} v^{\mathrm{b}} \mathrm{p}=0.17$

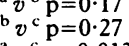

$v^{\mathrm{c}} \mathrm{p}=0.013$

$\mathrm{d} v \mathrm{e}=0.008$

$v \mathrm{p}=0.008$
$v \mathrm{p}=0.01$

$v^{\mathrm{f}} \mathrm{p}=0.77$

${ }_{\mathrm{h}} v_{\mathrm{i}}^{\mathrm{h}} \mathrm{p}=0 \cdot 25$

$\mathrm{g} v v_{\mathrm{i}=0.02}$
Unconjugated faecal bile acids

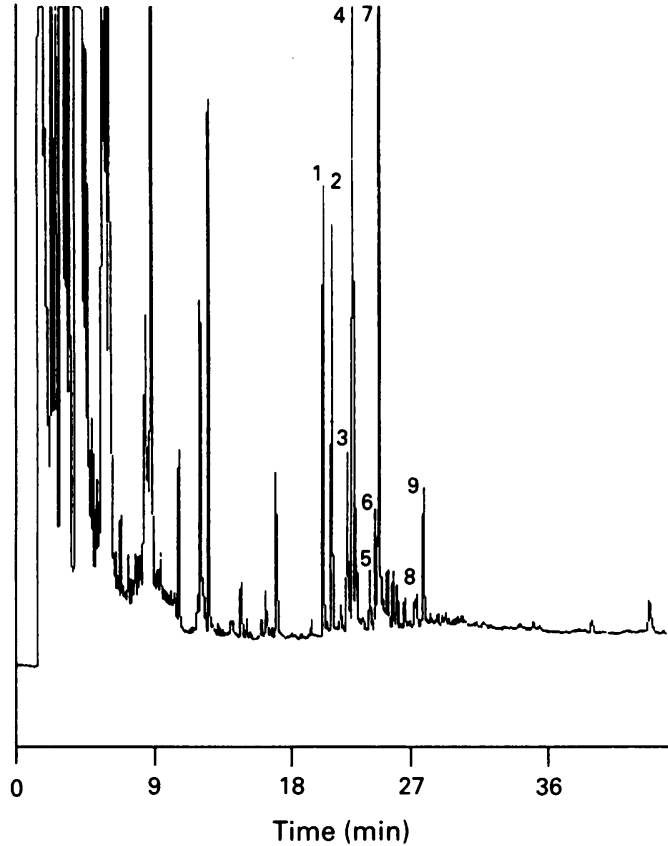

Unconjugated faecal bile acid profile.

$1=$ coprostanol $(G L C$ standard $)$

$2=$ nordeoxycholate (internal standard)

$3=3 \beta$ hydroxy- $5 \beta$ cholanoate (isolithocholate)

$4=3 \alpha$ hydroxy $-5 \beta$ cholanoate (lithocholate)

$5=3 \alpha, 12 \beta$ dihydroxy- $5 \beta$ cholanoate (isodeoxycholate)

$6=3 \alpha, 12 \beta$ dihydoxy-5 $\alpha$ cholanoate (allo-isodeoxycholate)

$7=3 \alpha, 12 \alpha$ dihydroxy- $5 \beta$ cholanoate (deoxycholate)

$8=3 \alpha, 7 \alpha, 12 \alpha$ trihydroxy-5 $\beta$ cholanoate (cholate)

$9=3 \alpha$ hydroxy-120xo-5 $\beta$ cholanoate (12-oxo-lithocholate)

acids excreted in faeces and are not further considered in this report.

The faecal bile acid concentration $(\mu \mathrm{mol} / \mathrm{g}$ dry weight) was greater in the polyp group when compared with controls. The daily faecal bile acid excretion was found to be significantly higher in the polyp group compared with cancer patients, while the difference between the polyp group and controls was of borderline significance (Table III)

Of the individual bile acids identified, a significantly lower concentration of the primary bile acid chenodeoxycholate was found in the faeces of cancer patients, indeed it was detected significantly less frequently in cancer patients (Table II). The concentration of lithocholic acid was found to be higher in patients with polyps than controls as was the concentration of total secondary bile acids (Table II).

TABLE III Concentration and daily excretion of faecal bile acids

\begin{tabular}{|c|c|c|c|}
\hline & $\begin{array}{l}\text { Controls } \\
(n=10)\end{array}$ & $\begin{array}{l}\text { Cancers } \\
(n=14)\end{array}$ & $\begin{array}{l}\text { Polyp } \\
(n=9)\end{array}$ \\
\hline $\begin{array}{l}\text { Bile acids ( } \mu \mathrm{mol} / \mathrm{g} \text { dry } \\
\text { weight faeces) } \\
\text { Bile acid daily } \\
\text { excretion }(\mu \mathrm{mol})\end{array}$ & $\begin{array}{c}1 \cdot 96^{\mathrm{a}} \\
0 \cdot 91-6 \cdot 97 \\
71 \cdot 1^{\mathrm{d}} \\
13 \cdot 4-303 \cdot 1\end{array}$ & $\begin{array}{l}3 \cdot 97^{\mathrm{b}} \\
0 \cdot 87-8 \cdot 41 \\
50 \cdot 6^{\mathrm{e}} \\
3 \cdot 5-209 \cdot 7\end{array}$ & $\begin{array}{l}5 \cdot 23^{\mathrm{c}} \\
2 \cdot 16-13 \cdot 67 \\
146 \cdot 5^{\mathrm{f}} \\
71 \cdot 8-327\end{array}$ \\
\hline
\end{tabular}

Upper figures indicate median values, lower figures in italics indicate range.

Kruskall-Wallis $(\mathrm{H}=6.77, \mathrm{df}=2) \quad \mathrm{a} v^{\mathrm{b}} v^{\mathrm{c}} \mathrm{p}=0.034$

Kruskall-Wallis $(\mathrm{H}=6.77, \mathrm{df}=2) \quad{ }^{\mathrm{d}} v^{\mathrm{e}} v^{\mathrm{f}} \mathrm{p}=0.045$

Mann Whitney ${ }^{\mathrm{a}} v^{\mathrm{b}} \mathrm{p}=\mathbf{0 . 3 3}$

$v^{\mathrm{c}} \mathrm{p}=0.016$

$v^{\mathrm{c}} \mathrm{p}=\mathbf{0 . 6 3}$

${ }_{v} v_{\mathrm{p}=0.067}^{\mathrm{p}=0.75}$

e $v$ f $\mathrm{p}=0.018$ 
TABLE IV Chenodeoxycholate (CDCA) and cholate (CA) derivatives (mol\%), ratios of $C D C A$ :total $C D C A$ derivatives and $C A$ :total $C A$ derivatives

\begin{tabular}{llll}
\hline & $\begin{array}{l}\text { Controls } \\
(n=10)\end{array}$ & $\begin{array}{l}\text { Cancers } \\
(n=14)\end{array}$ & $\begin{array}{c}\text { Polyps } \\
(n=9)\end{array}$ \\
\hline CDCA derivatives & $50 \cdot 27$ & $51 \cdot 84$ & $47 \cdot 08$ \\
& $44 \cdot 77-82 \cdot 48$ & $36 \cdot 68-93 \cdot 49$ & $38 \cdot 29-76 \cdot 64$ \\
CA derivatives & $49 \cdot 51$ & $45 \cdot 23$ & $52 \cdot 92$ \\
& $11 \cdot 04-55 \cdot 23$ & $6 \cdot 51-63.32$ & $23 \cdot 36-61 \cdot 32$ \\
CDCA:CDCA derivatives & $0 \cdot 052^{\mathrm{a}}$ & $0 \cdot 00^{\mathrm{b}}$ & $0 \cdot 042^{\mathrm{c}}$ \\
& $0-0 \cdot 18$ & $0-0 \cdot 04$ & $0-0 \cdot 21$ \\
CA:CA derivatives & $0 \cdot 037^{\mathrm{d}}$ & $0 \cdot 00^{\mathrm{e}}$ & $0 \cdot 032^{\mathrm{f}}$ \\
& $0-0 \cdot 17$ & $0-0 \cdot 04$ & $0-0 \cdot 23$ \\
\hline
\end{tabular}

Upper figures indicate medians, lower figures indicate range.

Kruskall-Wallis $(\mathrm{H}=9 \cdot 56, \mathrm{df}=2){ }^{\mathrm{a}} v^{\mathrm{b}} v^{\mathrm{c}} \mathrm{p}=0.009$

Kruskall-Wallis $(\mathrm{H}=6 \cdot 29, \mathrm{df}=2) \quad \mathrm{d}_{v^{\mathrm{e}}} v^{\mathrm{f}} \mathrm{p}=0.043$

Mann Whitney ${ }^{\mathrm{a}} v^{\mathrm{b}} \mathrm{p}=0.004$

$\mathrm{b} v^{\mathrm{c}} \mathrm{p}=0.008$

${ }^{\mathrm{a}} v^{\mathrm{c}} \mathrm{p}=0.9$

$\mathrm{d} v \mathrm{e}=0.034$

$\mathrm{d} v \mathrm{f} \mathrm{p}=0.97$

e $v_{\mathrm{p}} \mathrm{p}=0.034$

There were no significant differences in the proportions of total chenodeoxycholic acid or cholic acid derivatives between the three groups (Table IV). The proportion of metabolites of chenodeoxycholic acid and cholate was, however, higher in the cancer group, as reflected by lower ratios of chenodeoxycholic acid and cholate to their total derivatives (Table IV). The overal proportion of the secondary bile acids was highest among the cancer patients when compared with both polyp patients and controls (Table V). No significant differences were found in lithocholic acid:deoxycholic acid ratio or the lithocholic acid:deoxycholic acid $\times$ total faecal bile acid index when the groups were compared (Table V).

The proportion of secondary bile acids in each group was found to correlate strongly with both transit time and stool weight $\left(r_{s}=0.717, p<0.01\right.$ and $r_{s}=-0.527, p<0.01$ respectively)

\section{Discussion}

One of the key elements of this study was the exercise of tight control over variables which may influence faecal bile acid profiles; age, transit time, gall stones, cholecystectomy, hepatic function, hepatic metastases, previous surgery, and antibiotics.

Not only is there a significant day to day variation in faecal bile acid excretion, but faecal bile acid concentrations may vary between different areas of the same stool. ${ }^{46} \mathrm{~A}$ minimum complete three day stool collection with homogenisation has been shown to be necessary to minimise variation and most previous studies,

TABLE V Faecal bile acids: lithocholic acid: deoxycholic acid ratios, proportions of secondary bile acids

\begin{tabular}{lccc}
\hline Bile acid & $\begin{array}{l}\text { Controls } \\
(n=10)\end{array}$ & $\begin{array}{l}\text { Cancers } \\
(n=14)\end{array}$ & $\begin{array}{c}\text { Polyps } \\
(n=9)\end{array}$ \\
\hline LCA:DCA & $1 \cdot 49$ & $1 \cdot 84$ & $1 \cdot 31$ \\
LCA:DCA X TFBA & $0 \cdot 97-7 \cdot 47$ & $0 \cdot 97-14 \cdot 36$ & $0 \cdot 61-3 \cdot 43$ \\
$\% 2^{\circ}$ bile acids (mol\%) & $6 \cdot 21$ & $7 \cdot 9$ & $8 \cdot 19$ \\
& $1 \cdot 67-12 \cdot 41$ & $3 \cdot 76-19 \cdot 52$ & $2 \cdot 83-21 \cdot 91$ \\
& $95 \cdot 19^{\mathrm{a}}$ & $100^{\mathrm{b}}$ & $97 \cdot 19^{\mathrm{c}}$ \\
& $81 \cdot 73-100$ & $96 \cdot 85-100$ & $78 \cdot 18-100$ \\
\hline
\end{tabular}

Upper figures indicate median values, lower figures in italics indicate range.

LCA = lithocholic acid; DCA = deoxycholic acid; TFBA = total faecal bile acid concentration

$(\mu \mathrm{mol} / \mathrm{g})$.
Kruskall-Wallis $(\mathrm{H}=7 \cdot 1, \mathrm{df}=2) \quad{ }^{\mathrm{a}} v^{\mathrm{b}} v^{\mathrm{c}} \mathrm{p}=0.029$

Mann Whitney ${ }^{\mathrm{a}} v^{\mathrm{b}} \mathrm{p}=0.029$

$v^{\mathrm{c}} \mathrm{p}=0.02$

a $v$ c $\mathrm{p}=0.90$ with few exceptions, have not done this. In the present study complete five day stool collections were examined. Furthermore careful control was exercised over the collection of faeces, with immediate freezing of specimens to minimise the possibility of bacterial biotransformation of faecal bile acids.

Over a five day period patients with adenomatous polyps had a higher daily faecal bile acid excretion compared with cancers and higher total faecal bile acid and secondary bile. acid concentrations, compared with controls. Patients with established colorectal cancer showed increased proportions of secondary bile acids in their faeces.

Similar findings of an increased faecal bile acid concentration and increased secondary bile acid concentration in patients with adenomatous polyps were reported by Reddy and Wynder. ${ }^{26}$ Although stool collection in their study was only carried out for 24-48 hours, it was done at home. Breuer $e a^{36} l^{36}$ studied 12 patients with adenomatous polyps and found no differences in faecal bile acid excretion; the participants in this study were, however, hospitalised and a change in diet may be of relevance to their findings. Furthermore, it is not clear whether or not the control group was screened for polyps. Because polyps are a relatively common finding among a population of a similar age to their patients, ${ }^{50}$ this is a significant confounding factor. No attention was paid to previous cholecystectomy nor to the presence of gall stones. Both factors have been shown by several groups ${ }^{4251-53}$ to be associated with changes in biliary bile acid composition. Similar difficulties arise in the interpretation of another study of polyp patients that showed no differences in faecal bile acid excretion. ${ }^{35}$ Furthermore, this latter study was carried out on native Japanese, a population with a considerably lower incidence of colorectal cancer and probably not comparable with our population.

A significantly higher concentration of lithocholic acid was found in polyp patients compared with controls. This may be important as lithocholic acid has been shown to be genotoxic in in vitro studies. ${ }^{1+15}$

Patients with established colorectal cancer had a lower faecal concentration of the primary bile acid chenodeoxycholate and were found to excrete a higher proportion of secondary bile acids, compared with both controls and polyps. This was reflected by the increased proportions of the biotransformation products of both cholate and chenodeoxycholic acid seen, and is in accordance with two previous studies. ${ }^{26}{ }^{27}$ Our own recent studies ${ }^{+3}$ have shown an increased proportion of secondary biliary bile acids in patients with colorectal cancer. The changes we observed may have occurred as a result of an increased absorption of secondary bile acids, previously shown in patients with large and

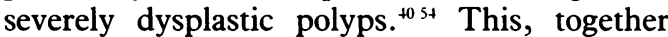
with feedback inhibition of primary bile acid synthesis, resulting from increased enterohepatic cycling of secondary bile acids, ${ }^{55}$ may explain the differences seen. In our studies therefore, faecal and biliary bile acid findings have been consistent. Although there were no differences in faecal bile acid concentration in those patients 
with colorectal cancer, total daily excretion was significently lower than in patients with polyps, an increased colonic absorption of bile acids may in part account for this. Further studies comparing the bile acid absorption in polyp patients with that in patients with established colorectal cancer, may help to clarify this issue.

A prolonged colonic transit time may be expected to result in increased bacterial metabolism of bile acids, and the observation that an increased proportion of secondary bile acids was strongly correlated with a prolonged bowel transit time is therefore of interest. Yet the cancer patients, who had significantly longer mouth-to-anus transit times, were not obstructed clinically or radiologically and stool frequencies were similar in all groups. Nagengast et $a l,{ }^{56}$ using a similar radioopaque marker method to our own, could find no relationship between faecal bile acid concentration or pattern and transit time in healthy controls. A recent study, ${ }^{57}$ however, showed an association between diets high in refined sugar and prolonged mouth-to-anus transit time (radioopaque markers), despite a reduced mouth-to-caecum transit time $\left(\mathrm{H}_{2}\right.$ breath test). This was coupled with an increased faecal bile acid concentration and proportion of secondary faecal bile acids. These findings would suggest that an interplay of various intraluminal factors, including bile acids themselves, may affect colonic function. Clearly the nature of this relationship requires further investigation, particularly as no other case controlled studies have reported on transit time in relation to faecal bile acid concentration.

As little is known about the distribution of bile acids between the water or solid fractions of faeces and their possible cellular effects, the observed differences in faecal bile acids may not totally reflect their significance. Indeed the presentation of bile acids to the colonic mucosa is likely to be dependant upon the concentration of bile acids in the water fraction of faeces. ${ }^{58}$ The role played by dietary fibre is of importance to this compartmentalisation because it binds bile acids. ${ }^{21}$ Moreover, the fermentation of fibre will have effects on colonic $\mathrm{pH} .{ }^{59}$ This may not only affect 7-alpha-dehydroxylase activity (responsible for secondary bile acid formation), ${ }^{60}$ but also the ionic composition and the solubility of bile acids in the water compartment of faeces. ${ }^{61}$ These factors and their interrelationships require elucidation in these patients. Nevertheless, we propose that the increased concentration of bile acids and increased daily excretion may predispose to the development of polyps. This could result from bile acid mediated effects on cellular proliferation through several possible pathways as already discussed. ${ }^{+16-18}$ The differences observed in cancer patients are more difficult to explain, as the faecal bile acid profiles of cancer patients in our study were different from those of polyps and controls. We would suggest that an altered metabolism (possibly related to gut transit time) may occur at the large polyp or cancer stage. This, with or without an increased absorption of faecal secondary bile acids may account for the differences observed in both faecal and biliary bile acids.
Neither the ratio of lithocholic acid: deoxycholic acid, or lithocholic acid:deoxycholic acid $\times$ total faecal bile acid were significantly increased in patients with colorectal cancer or polyps. This suggests that such indices are unlikely to provide useful markers for the identification of colorectal cancer risk as previously proposed..$^{37}$

In conclusion we have shown a significantly increased faecal bile acid concentration in patients with adenomatous polyps and an increased proportion of secondary bile acids in the faeces of patients with established colorectal cancer. This study lends further support to the theory that these compounds may have some role in the pathogenesis of polyps and colorectal cancer.

This project was funded by the Cancer Research Campaign to whom we are very grateful and included the salaried support of S Radley, C Imray, and A Davis. We are also grateful for additional financial support from the West Midlands Regional Health Authority (C Hendrickse is a Sheldon Research Fellow) and Dudley Road Hospital Surgical Research Fund and Cancer and Dudley Road Hospital Surgical Research Fund and Cance Research Fund. We are grateful to numerous colleagues in the
clinical departments of Dudley Road Hospital in particular the clinical departments of Dudley Road Hospital in pa
endoscopy, outpatient, day cases, and radiology units.

1 Armstrong B, Doll R. Environmental factors and cance incidence and mortality in different countries with special reference to dietary practices. Int $\mathcal{F}$ Cancer 1975; 15: 617-31.

2 Nicholson ML, Neoptolemos JP, Clayton HA, Heagerty AM Diet and colorectal cancer. Int Clin Nutr Rev 1988; 8 180-97.

3 Hill MJ. Microbes and human carcinogenesis. London: Edward Arnold, 1986: 62-107.

4 Cohen BI, Raicht RF, Deschner EE, Takahashi M, Sarwal AN, Fazzini E. Effect of cholic acid feeding on N-methyl-Nnitrosourea induced colon tumours and cell kinetics in rats. nitrosourea induced colon tumours

5 Narisawa T, Magadia NE, Weisburger JH, Wynder EL. Promoting effect of bile acids on colon carcinogenesis after intrarectal instillation of N-methy-N-nitro-N nitrosoguanidine in rats. $\mathcal{F}$ Natl Cancer Inst 1974; 53 1093-7.

6 Reddy BS, Watanabe K, Weisburger JH, Wynder EL. Promoting effect of bile acids in colon carcinogenesis in germ-free and conventional F 344 rats. Cancer Res 1977; 37 $3238-42$.

7 Wilpart M, Mainguet P, Maskens A, Roberfroid M. Structure activity relationship amongst bile acids showing comutagenic activity towards 1,2-dimethyl hydrazine. mutagenic activity towards

8 Watabe J, Bernstein $\mathrm{H}$. The mutagenicity of bile acids using a fluctuation test. Mutat Res 1985; 158: 45-51.

9 Venitt S, Bosworth D, Easton DF. Lack of mutagenic activity of bile acids in bacterial fluctuation tests. Mut Res 1987; 190 $190-6$.

10 Venitt S. Mutagens in human faeces and water of the large bowel. In: Roland IR (ed). Role of the gut flora in toxicity and cancer. London: Academic Press, 1988: 125-8.

11 Bernstein $\mathrm{H}$. The mutagenic activity of bile acids in fluctuation tests. Mut Res 1987; 191: 125-8.

12 Cheah PY, Bernstein H. Colon cancer and dietary fibre: cellulose inhibits the DNA-damaging ability of bile acids. Nutr Cancer 1990; 13: 51-7.

13 Russo $\mathrm{P}$, Taningher $M$, Pala $M$, Pisano V, Pedemonte $\mathbf{P}$ and hamster cells by lithocholic acid. Cancer Res 1987; 47: and hamster cells by lithocholic acid. Cancer Res 1987; 47: 2866-74.

14 Kulkarni MS, Cox BA, Yielding KL. Requirements of DNA strand breaks by lithocholic acid. Cancer Res 1982; 41: $2792-5$

15 Ferguson LR, Parry JM. Mitotic aneuploidy as a possible mechanism for tumour promoting activity in bile acids. Carcinogenesis 1984; 5: 447-52.

16 Bull AW, Marnett LJ, Dawe EJ, Nigro Nd. Stimulation of deoxythyridine incorporation in the colon of rats treated intrarectally with bile acids and fats. Carcinogenesis $1983 ; 4$ : 207-10.

17 Rampton DS, Breuer NF, Vaja SG, Sladen GE, Dowling RH Role of prostaglandins in bile-salt induced changes in rat Role of prostaglandins in bile-salt induced changes in

18 Fitzer CJ, O'Brien AO, Guillem JG, Weinstein IB. The regulation of protein kinase $\mathrm{C}$ by chenodeoxycholate, regulation of protein kinase $C$ by chenodeoxycholate,
deoxycholate and several structurally related bile acids. Carcinogenesis 1987; 8: 217-20.

19 Hill MJ, Drasar BS, Aries V, Crowther DS, Hawksworth G, Williams REO. Bacteria and aetiology of cancer of the large bowel. Lancet 1971; i: 95.

20 Reddy BS, Wynder EL. Large bowel carcinogenesis: Faecal constituents of populations with diverse incidence rates of colon cancer. F Natl Cancer Inst 1973; 50: 1437-42.

21 Setchell KDR, Street JM, Sjovall J. Fecal bile acids. In: Setchell KDR, Kritchevsky D, Nair PP, eds. The bile acids, chemistry, physiology and metabolism. Vol 4. Methods and applications. New York: Plenum Press, 1988: 441-575. 
22 Reddy BS. Diet and excretion of bile acids. Cancer Res 1981; 41: $3766-8$.

23 Crowther JS, Drasar BS, Hill MJ, Maclennan R, Magnin D Peach $S$, et al. Faecal steroids and bacteria and large bowel cancer in Hong Kong by socio-economic groups. Brf Cancer 1976; 34: 191-8.

24 Cummings JH, Wiggins HS, Jenkins DJA, Houston H, Jivra $\mathrm{T}$, Drasar BS, et al. Influence of diets high and low in anima fat on bowel habit, gastrointestinal transit time, faecal microflora, bile acid and fat excretion. F Clin Invest $1978 ; 61$ : microflora,

25 Van Fassen A, Bol J, Van Dokkum W, Pikaar N, Ockhuizen $\mathrm{T}$, Hermus RJJ. Bile acids, neutral steroids and bacteria in feces as affected by a mixed, a lacto-ovovegetarian and a vegan diet. Am F Clin Nutr 1987; 46: 962-7.

26 Reddy BS, Wynder EL. Metabolic epidemiology of colon cancer. Fecal bile acids and neutral sterols in colon cancer patients and patients with adenomatous polyps. Cancer 1977; 39: 2533-9.

27 Hill MJ, Drasar BS, Williams REO, Meade TW, Cox AG, Simpson JEP, et al. Faecal bile acids and Clostridia in patients with cancer of the large bowel. Lancet 1975; i: patients

28 IARC Intestinal Microecology Group. Dietary fibre, transi time, faecal bacteria steroids and colon cancer in two Scandinavian populations. Lancet 1977; ii: 207-11.

29 Murray WR, Blackwood A, Mackay C, Calman K. Faecal bile acids and clostridia in the aetiology of colorectal cancer and breast cancer. $B r \mathcal{F}$ Cancer 1980; 41: 923-8.

30 Moskovitz M, White C, Barnett RN, Stevens S, Russell E, Vargo D, et al. Diet, faecal bile acids and neutral sterols in carcinoma of the colon. Dig Dis Sci 1979; 24: 746-51

31 Mudd DG, McKelvey STD, Norwood W, Elmore DT, Roy AD. Faecal bile acid concentrations in patients with carcinoma or at increased risk of carcinoma of the large bowel. Gut 1980; 21: 587-90.

32 Kaibara N, Sasaki T, Ikeguchi M, Kog AS, Ikawa S. Faeca bile acids and neutral sterols in Japanese with large bowel bile acids and neutral sterols in Japant

33 Hikasa Y, Tanida M, Ohno T, Shimoyama T. Faecal bile acid profiles in patients with large bowel cancer in Japan. Gu 1984; 25: 833-8.

34 Breuer NF, Dommes P, Jaekel S, Goebell H. Faecal bile acid excretion pattern in colonic cancer patients. Dig Dis $S c$ 1985; 30: 852-9.

35 Tanida N, Hikasa Y, Shimoyama T, Setchell KDR. Comparison of faecal bile acid profiles between patients with adenomatous polyps of the large bowel and healthy subjects in Japan. Gut 1984; $25: 824-32$.

36 Breuer NF, Jaekel S, Dommes P, Goebell H. Faecal bile acids in patients with adenomatous polyps of the colon. Digestion 1986; 34: 87-92

37 Owen RW, Henly PJ, Thompson MH, Hill MJ. Steroids and cancer: faecal bile acid screening for early detection of cance risk. F Steroid Biochem 1986; 24: $391-4$.

38 Owen RW, Dodo M, Thompson MH, Hill MH. Faeca steroids and colorectal cancer. Nutr Cancer 1987; 9: 73-80.

39 Hofmann AF. Chemistry and enterohepatic circulation of bile acids. Hepatology 1984; 4: 4-14s.

40 van der Werf SDJ, Nagengast FM, van Berge Henegouwen GP, Huijbregts AWM, van Tongeren JHM. Colonic absorption of secondary bile acids in patients with adenomatous polyps and in matched controls. Lancet 1982; i: 759-62.

41 Moorehead RJ, Campbell GR, Donaldson JD, McKelvey STD. Relationship between duodenal bile acids and STD. Relationship between duodenal

42 Castleden WM, Detchon P, Misso NLA. Biliary bile acids in cholelithiasis and colon cancer. Gut 1989; 30: 860-5.
43 Radley S, Imray CHE, Davis AE, Hendrickse C, Lawson A, Baker PR, et al. Duodenal gallbladder bile acid profiles in patients with colorectal cancer or polyps [Abstract] BrF Surg 1991; 78: 1497 .

44 Hinton JM, Lennard Jones JE, Young AC. A new method for studying gut transit times using radioopague markers. $G u t$ 1969; 10: 842-7.

45 Setchell KDR, Lawson AM, Tanida M, Sjovall J. General methods for the analysis of metabolic profiles of bile acids and related compounds in faeces. F Lipid Res 1983; 24 : 1085100 .

46 Setchell KDR, Ives JA, Cashmore GC, Lawson AM. On the homogenicity of stools with respect to bile acid composition and normal day-to-day variations: a detailed qualitative and quantitative study using capillary column gas chromatography - mass spectrometry. Clin Chim Acta 1987; 162 257-74.

47 Imray CHE, Davis A, Barker G, Radley S, Baker PR, Neoptolemos JP. Improved human faecal bile acid extraction. Biochem Soc Trans 1991; 19: 201S.

48 Almé BA, Bremmelgaard A, Sjovall J, Thomassen P. Analysis of metabolic profiles of bile acids in urine using a lipophillic anion exchanger and computerised gas-liquid chromatoanion exchanger and computerised gas-liquid chromato-

49 Lawson AM, Setchell KDR. Mass spectrometry of bile acids. In: Setchell KDR, Kritchevsky D, Nair PP, eds. The bile acids, chemistry, physiology and metabolism. Vol 4 methods and applications. New York: Plenum Press, 1988 $167-267$

50 Williams AR, Balasooriya BAW, Day DW. Polyps and cancer of the large bowel: A necropsy study in Liverpool. Gut 1982 23: $835-42$.

51 Malagelada JR, Go VLW, Summerskill HJ, Gamble WS Bileacid secretion and biliary bile acid composition altered by cholecystectomy. Dig Dis $1973 ; 18: 455-9$.

52 Hepner G, Hofman A, Malagelada J, Szczepanik P, Klein P. Increased bacterial degradation of bile acids in cholecystectomized patients. Gastroenterology 1974; 66: 556-64

53 Fisher MM, Yousef IM. Sex differences in the bile acid composition of human bile: studies in patients with and composition of human bile: studies in patients with
without gallstones. Can Med Ass F 1973; 109: 190-3.

54 van der Werf SDJ, Nagengast FM, van Berge Henegouwen GP, Huijbregts AWM, van Tongeren JHM. Intracolonic environment and the presence of colonic adenomas in man. Gut 1983; 24: 876-80.

55 Redinger R. The economy of the enterohepatic circulation of bile acids in the baboon. 2 . Regulation of bile acid synthesis by enterohepatic circulation of bile acids. $\mathcal{F}$ Lipid Res 1984 25: $437-47$.

56 Nagengast FM, van der Werf SDJ, Lamers HLM, Hector MPC, Buys WCAM, van Tongeren TMH. Influence of age, intestinal transit time and dietary composition on faecal bile intestinal transit time and dietary composition on faecal bile

57 Kruis W, Forstmaier G, Schewlen C, Stallard F. Effects of diets low and high in refined sugars on gut transit, bile acid metabolism and bacterial fermentation. Gut 1991; 32: 367-71

58 Rafter JJ, Child P, Anderson AM, Alder R, Eng V, Bruce WR Cellular toxicity of human faecal water depends on diet. Am F Clin Nutr 1987; 45: 559-63.

59 Thornton JR. High colonic $\mathrm{pH}$ promotes colorectal cancer. Lancet 1981; i: 1081-3.

60 Aries V, Hill MJ. Degradation of steroids by intestinal bacteria II. Biochim Biophys Acta 1970; 202: 535-43.

61 Van der Meer R, Welberg JWM, Kulpers F, Kleibeuker JH Mulder NH, Termont DSML, et al. Effects of supplemental dietary calcium on the intestinal association of calcium phosphate, and bile acids. Gastroenterology 1990; 99: 1653-9. 